ler: St. Jude Medical, Inc.), das durch Erzeugung eines künstlichen Pulses ständig durchgespült wird und dadurch weniger anfällig für Thrombenbildung ist.

Die Zentrifugalpumpe mit magnetisch gelagertem Rotor wurde erstmals 2014 in der MHH implantiert (Schmitto JD et al. J Heart Lung Transplant. 2015; 34(6):858-60). Die Pumpe verfügt über texturierte Blutkontaktflächen: Das Blut kommt, wie auch im Herzen, nur mit Gewebe und nicht mit künstlichen Materialien in Berührung. Der Patient trägt einen externen Controller und ein Akkusystem, das die Pumpe betreibt. Das HeartMate $3^{\text {tx }}$ kann bis zu zehn Liter Blut pro Minute und damit die gesamte Blutmenge eines gesunden Herzens pumpen. Bisher wurden an der MHH acht Patienten mit dem neuen Gerät versorgt; bei keinem sind perioperativ schwerwiegende Komplikationen aufgetreten. Die 1-Jahres-Überlebensrate beträgt $81 \%$.

Derzeit tragen rund 700 Patienten das HeartMate $3^{\mathrm{m}}$, mit dem bisher keinerlei Pumpenthrombosen aufgetreten sind.

Noch in der klinischen Entwicklung ist die MVAD ${ }^{\circledR}$-Pumpe (Hersteller: Heart-
Ware International, Inc.). Diese Pumpe ist weniger als halb so groß wie die HVAD ${ }^{\circledR}$ Pumpe - derzeit das kleinste kommerziell erhältliche Vollunterstützungsimplantat - und wird mit einer weniger invasiven Thorakotomietechnik eingesetzt. Sie besitzt einen steuerbaren Nahtring, mit dem der Arzt die Einflusskanüle für optimalen Blutfluss in die Pumpe positionieren kann. Zudem sollen die Steuereinheit und die Batterieeinheit zum MVAD ${ }^{\circledR}$ die Benutzung deutlich verbessern, u.a. ist die Driveline dünner. Die laufende $\mathrm{Zu}$ lassungsstudie für die $\mathrm{MVAD}^{\circledR}$-Pumpe wurde wegen Problemen mit der Controller-Einheit zunächst unterbrochen.

\section{Ziel: Transplantation übertrumpfen}

Die nächste Generation der VAD-Systeme dürfte noch kleiner werden, um die Implantierbarkeit weiter zu erleichtern. Die Controller sollen leichter und die Lithiumbatterien stärker werden. Das ermöglicht eine drahtlose, netzwerkgestützte Fernüberwachung der Patientendaten rund um die Uhr, wie dies bereits beim HeartAssist $5^{\circledR}$-System (Hersteller: ReliantHeart, Inc.) möglich ist. Zudem wird weiterhin an vollimplantierbaren Systemen mit transkutaner Energiezufuhr gearbeitet - sie kämen folglich ohne Driveline aus und könnten das Infektionsrisiko erheblich verringern. Diese Systeme, von denen noch keines Marktreife erlangt hat, könnten sich zu einer echten „destination therapy“ entwickeln, da sie womöglich für den Patienten langfristig vorteilhafter sind als eine Herztransplantation.

Die MHH hat maßgeblich die Entwicklung minimalinvasiver Verfahren zur VAD-Implantation vorangetrieben (Schmitto JD et al. J Thorac Cardiovasc Surg. 2012;143:511-13) und praktiziert daher vorzugsweise den schonenden $\mathrm{Zu}$ gang via anterolateraler Thorakotomie und oberer Hemisternotomie. Minimalinvasiv operierte Patienten haben kleinere OP-Narben, einen verkürzten Klinikaufenthalt und ein verringertes Infektionsrisiko. Ob sich das Outcome der Patienten - das 1-Jahres-Überleben mit nonpulsatilen intrakorporalen LVAD liegt derzeit bei $80 \%$, das 2-Jahres-Überleben bei $70 \%$ - so weiter verbessern lässt, bleibt abzuwarten. Dr. Beate Grübler

\title{
ECMO und VAD sind immer länger im Einsatz
}

ECMO und VAD wurden früher nur kurzzeitig zur Überbrückung bis zu einer Lungen- bzw. Herztransplantation eingesetzt. Doch die Weiterentwicklung der Geräte schreitet voran und damit auch deren Einsatzmöglichkeiten, wie Prof. Axel Haverich von der medizinischen Hochschule Hannover erläutert.

? Herr Prof. Haverich, die Anzahl an ECMO-Patienten in der MHH konnte in den letzten Jahren kontinuierlich gesteigert werden. Die Miniaturisierung der ECMOPumpensysteme, die Verbesserung der Pumpentechnik sowie die Optimierung der Gasaustauschmembranen und Kathetertechnologie haben das Verfahren effektiver und sicherer gemacht. Wie lange können heute Patienten mit dem extrakorporalen Lungenersatz versorgt werden?

Haverich: Wir können Patienten heute für mehrere Monate an der ECMO halten, derzeit jedoch noch unter intensivmedizinischen Bedingungen. Die stetig wachsende Zahl von Patienten mit ECMO-Behand- lung zeigt, dass noch nicht alle behandelt werden, die davon profitieren könnten. Das gilt gleichermaßen für Patienten nach herz- oder lungenchirurgischen Eingriffen wie auch für jene mit akutem Lungenversagen, das sich besonders häufig auf dem Boden einer Viruspneumonie entwickelt.

? Bei den VAD-Systemen gab es ebenfalls deutliche technische Fortschritte, wodurch u.a. das Risiko von Pumpenthrombosen reduziert wurde. Könnte dadurch auch der Bedarfan Antikoagulanzien sinken und die Nachsorge neu konzipiert werden?

Haverich: Ja, deshalb bemühen sich unsere
Klinik wie auch andere herzchirurgische Zentren um eine Einstellung der Antikoagulation, um einerseits Blutungskomplikationen beim Patienten und andererseits Gerinnungsphänomene im VAD-System zu vermeiden - das muss unter Abwägung der Risiken allerdings mit aller Vorsicht erfolgen.

? Sie setzen bei der VAD-Implantation auf minimalinvasive Verfahren. Worin liegt der entscheidende Vorteil und wird dieser in den Leitlinien berücksichtigt? Haverich: Wir Chirurgen fokussieren uns ja nicht so sehr auf Guidelines, insbesondere wenn es sich um neuere Verfahren und solche in noch intensiver klinischer Erprobung handelt. Auch ohne Leitlinien verbreitet sich das von uns entwickelte Verfahren rasant im internationalen Raum, weil die Patienten sich rascher erholen und weniger perioperative Komplikationen haben. Interview: Beate Grübler 